ILD. The $80 \%$ cut-off for $\%$ DLco may be proposed to identify isolated vascular involvement, while $\% \mathrm{FVC}$ at $80 \%$ or $\% \mathrm{DLco}$ at $70 \%$ to identify significant parenchymal involvement. These results need to be confirmed in larger multi-centric cohorts.

Disclosure of Interests: Cosimo Bruni Speakers bureau: Actelion, Eli Lilly, Mariaelena Occhipinti Consultant of: Imbio, Gianna Camiciottoli: None declared, Maurizio Bartolucci: None declared, Gemma Lepri: None declared, Alessio Fabbrizzi: None declared, Alessandra Tottoli: None declared, Anna Bassetto: None declared, Giuglia Ciardi: None declared, Dilia Giuggioli: None declared, Giovanna CUOMO: None declared, Francesco Masini: None declared, Federico Lavorini: None declared, Linda Calistri: None declared, Marco Matucci-Cerinic Grant/research support from: Actelion, MSD, Bristol-Myers Squibb, Speakers bureau: Acetelion, Lilly, Boehringer Ingelheim DOI: 10.1136/annrheumdis-2020-eular.2188

\section{OP0182 \\ PROTEOGLYCAN LOSS IN ARTICULAR CARTILAGE IS ASSOCIATED WITH JOINT INFLAMMATION SEVERITY IN PSORIATIC ARTHRITIS - A COMPOSITIONAL MAGNETIC RESONANCE IMAGING STUDY}

P. Sewerin ${ }^{1}$, D. Abrar ${ }^{2}$, S. Nebelung ${ }^{2}$, M. Frenken ${ }^{2}$, T. Ulrich ${ }^{2}$, K. L. Radke ${ }^{2}$, G. Antoch ${ }^{2}$, S. Vordenbäumen ${ }^{1}$, R. Brinks ${ }^{1}$, M. Schneider ${ }^{1}$, B. Ostendorf ${ }^{1}$, C. Schleich ${ }^{2}{ }^{1}$ Heinrich-Heine University, Department for Rheumatology, Duesseldorf, Germany; ${ }^{2}$ Heinrich-Heine University, Institut for Diagnostic and Interventional Radiology, Duesseldorf, Germany

Background: Even though cartilage loss is a known feature of psoriatic arthritis (PsA), little is known about its role in the pathogenesis of PsA. Using delayed gadolinium-enhanced magnetic resonance imaging of cartilage (dGEMRIC) as a non-invasive marker of the tissue's proteoglycan content, such early (i.e. pre-morphological) changes have been associated with inflammation in rheumatoid arthritis (RA). Yet, this association has not been studied before in PsA Objectives: Is the severity of local joint inflammation associated to local proteoglycan loss in PsA patients?

Methods: Metacarpophalangeal (MCP), proximal interphalangeal (PIP), and distal interphalangeal (DIP) joints of 17 patients with active PSA were evaluated by high-resolution clinical standard morphological and dGEMRIC sequences using a 3T MRI scanner (Magnetom Skyra, Siemens) and a dedicated 16-channel hand coil. Images were analyzed by two independent raters for dGEMRIC indices, PsA MRI scores (PsAMRIS) and total cartilage thickness (TCT). Kendall-Tau correlation coefficients $(\tau)$ were calculated.

Results: We found significant negative correlations between dGEMRIC indices and total PSAMRIS $(\tau=-0.5, p=0.012)$, synovitis $(\tau=-0.56, p=0.006)$, flexor tenosynovitis $(\tau=-0.4, p=0.049)$, and periarticular inflammation $(\tau=-0.72, p<$ 0.001 ). Significant positive correlations were found between TCT and dGEMRIC indices in all joint levels $(\tau=0.43, p<0.001)$. No significant correlations were determined between dGEMRIC indices and bone erosion, bone edema or bone proliferation.

Conclusion: In PsA, proteoglycan loss as assessed by dGEMRIC is associated with periarticular inflammation, synovitis, and flexor tenosynovitis, but not with bone erosion or proliferation, thereby highlighting the need for effective anti-inflammatory treatment regimes. Beyond morphology, advanced MRI techniques may be used to assess cartilage composition in PsA and to identify early

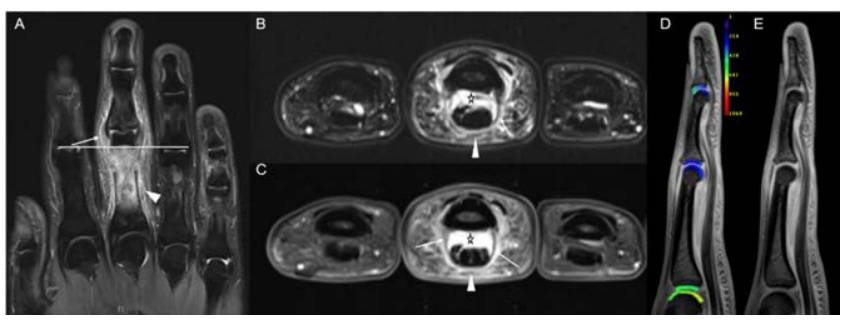

Figure 1 Right hand of a 26-year-old male with psoriatic arthritis Coronal STIR image (A) of digits 1-5, transversal fat-saturated (fS) T2-weighted image of digits 2-4 (B) and the corresponding transversal fs contrast-enhanced T1-weighted image (C) at the distal portion of the proximal phalanges. Horizontal white bar in (A) indicates level of transversal slices (B) \& (C). Sagittal fs Proton Density-weighted image of the third digit (D). A: Increased signal at the collateral ligaments and synovitis of the proximal interphalangeal (PIP) joint of the third digit (white arrow). Periarticular inflammation around the PIP joint and the body of the proximal phalanx of the third digit (arrowhead). B \& C: Extensive flexor tenosynovitis (asterix) and periarticular inflammation in the subcutaneous tissues (arrowhead) alongside thickened flexor tendon pulleys (arrow). D \& E: Representative sagittal T1-weighted images of the MCP, PIP and DIP joint of the 3rd digit. Following iv contrast administration and appropriate delay of $40 \mathrm{~min}$, A gives the morphologicalT1 map, while B gives the corresponding parameter map with dGEMRIC values [ms] overlaid. Note the significant decrease in dGEMRIC indices of the PIP joint as compared to the MCP joint. changes in cartilage as an imaging biomarker with potential application in detection and monitoring of PsA.

Disclosure of Interests: Philipp Sewerin Grant/research support from: AbbVie Deutschland GmbH \& Co. KG

Bristol-Myers Squibb Celgene $\mathrm{GmbH}$

Lilly Deutschland GmbH

Novartis Pharma GmbH Pfizer Deutschland GmbH

Rheumazentrum Rhein-Ruhr, Consultant of: AMGEN GmbH AbbVie Deutschland $\mathrm{GmbH} \&$ Co. KG Biogen GmbHBristol-Myers Squibb Celgene $\mathrm{GmbH}$ Chugai Pharma arketing Ltd. / Chugai Europe GmbHHexal Pharma Janssen-Cilag$\mathrm{GmbH}$ Johnson \& Johnson Deutschland GmbHLilly Deutschland GmbH / Lilly Europe / Lilly Global Novartis Pharma GmbH Pfizer Deutschland GmbH Roche Pharma Rheumazentrum Rhein-Ruhr Sanofi-Genzyme Deutschland GmbH Swedish Orphan Biovitrum GmbH UCB Pharma $\mathrm{GmbH}$, Speakers bureau AMGEN GmbH AbbVie Deutschland GmbH \& Co. KG Biogen GmbHBristol-Myers Squibb Celgene GmbH Chugai Pharma arketing Ltd. / Chugai Europe GmbHHexal Pharma Janssen-CilagGmbH Johnson \& Johnson Deutschland GmbHLilly Deutschland GmbH / Lilly Europe / Lilly Global Novartis Pharma $\mathrm{GmbH}$ Pfizer Deutschland GmbH Roche Pharma Rheumazentrum Rhein-Ruhr Sanofi-Genzyme Deutschland $\mathrm{GmbH}$ Swedish Orphan Biovitrum GmbH UCB Pharma GmbH, Daniel Abrar: None declared, Sven Nebelung: None declared, Miriam Frenken: None declared, Tim Ulrich: None declared, Karl Ludger Radke: None declared, Gerald Antoch: None declared, Stefan Vordenbäumen: None declared, Ralph Brinks: None declared, Matthias Schneider Grant/research support from: GSK, UCB, Abbvie, Consultant of: Abbvie, Alexion, Astra Zeneca BMS, Boehringer Ingelheim, Gilead, Lilly, Sanofi, UCB, Speakers bureau: Abbvie, Astra Zeneca, BMS, Chugai, GSK, Lilly, Pfizer, Sanofi, Benedikt Ostendorf: None declared, Christoph Schleich: None declared DOI: 10.1136/annrheumdis-2020-eular.5557

\section{OP0183 DO CARTILAGE LAMINAR COMPOSITIONAL CHANGES AS ASSESSED BY T2 RELAXOMETRY PREDICT INCIDENT AND WORSENING OF STRUCTURAL MORPHOLOGIC DAMAGE IN THE SAME PLATE 3 YEARS LATER?}

F. Roemer $^{1,2}$, F. Eckstein ${ }^{3,4}$, G. Duda ${ }^{5}$, S. Maschek ${ }^{3,4}$, A. Guermazi ${ }^{2,6}$, W. Wirth ${ }^{3,4}$. 'Universitätsklinikum Erlangen, Erlangen, Germany; ${ }^{2}$ Boston University, Boston, United States of America; ${ }^{3}$ Paracelsus Medical University, Salzburg, Austria; ${ }^{4}$ Chondrometrics $\mathrm{GmbH}$, Ainring, Germany; ${ }^{5}$ Charité, Berlin, Germany; ${ }^{6}$ VA Boston Healthcare System, Boston, United States of America

Background: To address the question whether laminar changes in knee cartilage T2 are relevant for prediction of lesion onset or progression in the same articular plate we included two different samples from the Osteoarthritis Initiative (OAI) study without radiographic osteoarthritis (ROA), i.e. so-called "healthy controls" with no ROA in either knee and being free of risk factors, and those with $\mathrm{K}-\mathrm{L} 0$ in one knee and ROA in the contralateral knee. Given the concept of the osteochondral unit, we hypothesize that superficial T2 is elevated in cartilage plates with subsequent surface damage development or worsening and deep layer T2 is elevated for those with subsequent bone marrow lesion (BML) development or worsening.

Objectives: To analyze whether knees with subsequent morphologic cartilage and BML development or worsening exhibit elevated cartilage T2 compared to those that do not develop such structural damage in the same plate 3 years later.

Methods: We included 63 knees from the OAI without ROA (K-L 0), but with definite $R O A(K-L \geq 2)$ in the contralateral knee, and 78 participants from the OAI healthy reference cohort.

Cartilage integrity or damage and subchondral bone marrow lesions (BMLs) were assessed for year 1 (i.e. baseline (BL) in this analysis) and year 4 (Y4) in chronological order using the semi-quantitative MOAKS scoring system.

$\mathrm{BL}$ deep and superficial layer cartilage T2 was computed from sagittal multi-echo spin echo MR images. Because cartilage T2 is known to display spatial variation with tissue depth, the segmented cartilages were computationally divided into superficial and deep $50 \%$, based on the distance between the segmented cartilage surface and bone interface. Statistical analyses were performed for the femoro-tibial (FT) joint on a plate level, i.e. medial femur (MF), medial tibia (MT) lateral femur (LF) and lateral tibia (LT), using UNIANOVA with adjustment for age, body mass index, sex, and sample.

Results: 141 participants were included. Of these 79 (56\%) were women, had a mean age of $59.4 \pm 9.1$ years and a mean body mass index of $25.8 \pm 4.1$ $\mathrm{m} / \mathrm{kg}^{2}$.

$52(37 \%)$ had prevalent cartilage lesions in the medial FT joint and $67(48 \%)$ in the lateral FT joint. For BMLs these numbers were 15 (11\%) medially and $14(10 \%)$ laterally. Worsening of FT cartilage lesions from BL to Y4 were seen in $10(7 \%)$ medially and $21(15 \%)$ in the lateral FT compartment. Incident FT cartilage lesions were seen in $11(11.5 \%)$ medially and 8 knees laterally. No worsening BMLs were seen 
medially and 2 knees showed worsening BMLs laterally. 10 (7\%) knees showed incident BMLs medially and $8(6 \%)$ knees in the lateral FT compartment.

Deep layer T2 showed prolongation in the LT in knees with incident LT cartilage lesions ( $n=8,34.5$ vs. $32.7 \mathrm{~ms}, \mathrm{p}=0.02$ ) and for MF in knees with MF cartilage lesion worsening ( $n=9,47.6$ vs. $41.4 \mathrm{~ms}, p=0.01)$ and MF BML incidence $(n=6$, 45.4 vs. $41.6 \mathrm{~ms}, \mathrm{p}=0.000$ ). Superficial T2 showed prolongation in the MT only in those knees with MT cartilage lesion worsening ( $n=2,47.3$ vs. $43.4 \mathrm{~ms}, p=0.03$ ). No additional associations were seen for the superficial layer.

Conclusion: For knees without ROA, BL deep layer T2 prolongation was seen for those who developed incident cartilage damage in the LT, and those with worsening cartilage damage and incident BMLs in the MF, respectively. Superficial T2 showed prolongation only in the MT for those with MT cartilage lesion worsening. In summary and contrary to our hypothesis the deep cartilage layer seems to be more relevant for cartilage damage development or worsening in the same FT plate than the superficial layer.

Acknowledgment: German Bundesministerium für Bildung und Forschung (BMBF - 01EC1408D -OVERLOAD-PREVOP)

Disclosure of Interests: Frank Roemer: None declared, Felix Eckstein Grant/ research support from: Merck, Orthotrphix, Servier, Galapagos, Kolon Tissuegene, Samumed, Novartis, Consultant of: Merck, Bioclinica, Servier, Samumed, Roche, Kolon Tissuegene, Galapagos and Novartis, Employee of: co-owner and employment with Chondrometrics, Georg Duda: None declared, Susanne Maschek Shareholder of: Stock/stock options at Condrometrics $\mathrm{GmbH}$, Employee of: Employment at Condrometrics $\mathrm{GmbH}$, Ali Guermazi Consultant of: AventisGalapagos, Pfizer, Roche, AstraZeneca, Merck Serono, and TissuGene, Wolfgang Wirth: None declared

DOI: 10.1136/annrheumdis-2020-eular.1813

\section{Osteoarthritis: clinical}

\section{OP0184 RISK OF COMORBIDITIES FOLLOWING INCIDENT CLINICIAN-DIAGNOSED KNEE OR HIP OSTEOARTHRITIS: A REGISTRY-BASED COHORT STUDY}

K. Pihl ${ }^{1}$, A. Turkiewicz ${ }^{1}$, V. Hughes ${ }^{1}$, W. Zhang ${ }^{2}$, S. M. A. Bierma-Zeinstra ${ }^{3}$, D. Prieto-Alhambra ${ }^{4}$, M. Englund ${ }^{1} .{ }^{1}$ Lund Univ., Lund, Sweden; ${ }^{2}$ Univ. of Nottingham, Nottingham, United Kingdom; ${ }^{3}$ Univ. Med. Ctr. Rotterdam, Rotterdam, Netherlands; ${ }^{4}$ Univ. of Oxford, Centre for Statistics in Medicine, NDORMS, Oxford, United Kingdom

Background: Osteoarthritis $(\mathrm{OA})$ is associated with increased comorbidity, but knowledge of the temporal relationships between $\mathrm{OA}$ and comorbidities is limited. Objectives: To estimate the risk of consulting for comorbidities in people with incident clinician-diagnosed knee or hip OA.

Methods: Using the Swedish Population Register, we identified all residents aged 35 years or above in the Skåne region, Sweden (total pop. 1.23 million) in the year 2009. We used the Skåne Health Care Register (SHR), a comprehensive register based on physicians' International Classification of Disease (ICD) 10, to identify persons who had at least one diagnosis of knee or hip OA - the exposures of interest - and 18 other diagnoses (Figure 1) - the outcomes of interest - between 1 January 2010 and 31 December 2017. To minimize potential confounding due to propensity to seek care, persons had to have at least one health-care visit with any diagnosis registered between 1 January 1998 and 31 December 2009 to be included. We excluded persons with a knee or hip OA diagnosis before 1 January 2010, and for each analysis, persons with a previous diagnosis of the outcome diagnosis of interest. People were followed from 1 January 2010 until relocation outside of the region, death, outcome diagnosis, or 31 December 2017, whichever came first. We calculated hazard ratios (HR) of each outcome diagnosis using Cox proportional hazard models with incident knee and hip OA as time-varying exposure. Time from start of follow-up to the date of an OA diagnosis was treated as unexposed, while time from an OA diagnosis to the outcome diagnosis was treated as exposed. Models were adjusted for residential area, if born in Sweden, annual income, years of education, marital status, sex, and age (all retrieved from Statistics Sweden), and baseline conditions (i.e. the other outcome diseases of interest, and alcohol-related disorders) as registered in SHR before 1 January 2010

Results: We included 548,681 persons in the Skåne population having at least one health-care visit during 1998 to 2009 and no doctor-diagnosed knee or hip OA. Of these, 36,465 persons consulted for knee OA and 14,477 for hip OA during the follow-up period (Table 1). Persons with clinician-diagnosed incident knee or hip OA have $8-61 \%$ higher hazard of depression, cardiovascular diseases, back pain, and osteoporosis than persons without an OA diagnosis (Fig. 1). Both knee and hip OA persons have increased risk of diabetes, however the $95 \%$ confidence interval excluded 1 only for knee OA (Fig. 1). Also, only knee OA patients have an increased risk of fracture to the forearm. For the rest of the diagnoses, we found either no increased risk for knee or hip OA persons, or estimates with wide confidence intervals, excluding any clear interpretations of the direction or size of the effects.

Table 1. Demographics of the study cohort at start of follow-up (Jan. 1, 2010).

\begin{tabular}{|c|c|c|c|}
\hline & $\begin{array}{c}\text { No OA } \\
(n=497,739)\end{array}$ & $\begin{array}{c}\text { Incident } \\
\text { knee OA } \\
(n=36,465)\end{array}$ & $\begin{array}{c}\text { Incident } \\
\text { hip OA } \\
(\mathrm{n}=14,477)\end{array}$ \\
\hline Age, yrs (SD) & $57.3(14.6)$ & $62.2(12.2)$ & $65.3(11.7)$ \\
\hline Women, n (\%) & $254,593(51)$ & $21,553(59)$ & $8,306(57)$ \\
\hline Married $^{\mathrm{a}}, \mathrm{n}(\%)$ & $350,172(70)$ & $27,955(77)$ & $10,937(76)$ \\
\hline Born in Sweden ${ }^{\mathrm{b}}, \mathrm{n}(\%)$ & $422,713(85)$ & $31,292(86)$ & $12,949(89)$ \\
\hline \multicolumn{4}{|l|}{ Education $^{\mathrm{c}}, \mathrm{n}(\%)$} \\
\hline$<10 \mathrm{yrs}$ & $128,738(26)$ & $11,364(31)$ & $4,745(33)$ \\
\hline $10-12$ yrs & $222,147(45)$ & $16,390(45)$ & $6,050(42)$ \\
\hline $13-14$ yrs & $59,950(12)$ & $3,908(11)$ & $1,556(11)$ \\
\hline$\geq 15 \mathrm{yrs}$ & $83,620(17)$ & $4,590(13)$ & 2,065 (14) \\
\hline Income in 100,000 SEK $^{d}$, mean (SD) & $2.3(3.0)$ & $2.1(2.4)$ & $2.1(2.6)$ \\
\hline
\end{tabular}

Missings: ${ }^{\mathrm{a}} 29^{\mathrm{b}} 44,{ }^{\mathrm{c}} 3,558,{ }^{\mathrm{d}} 2$

Conclusion: Incident clinician-diagnosed knee and hip $\mathrm{OA}$ are associated with increased risk of consultation for depression, cardiovascular disease, back pain osteoporosis, and diabetes. Results support previous findings for cardiovascula diseases and diabetes, however suggesting that the risk of diabetes is mainly associated with knee OA.

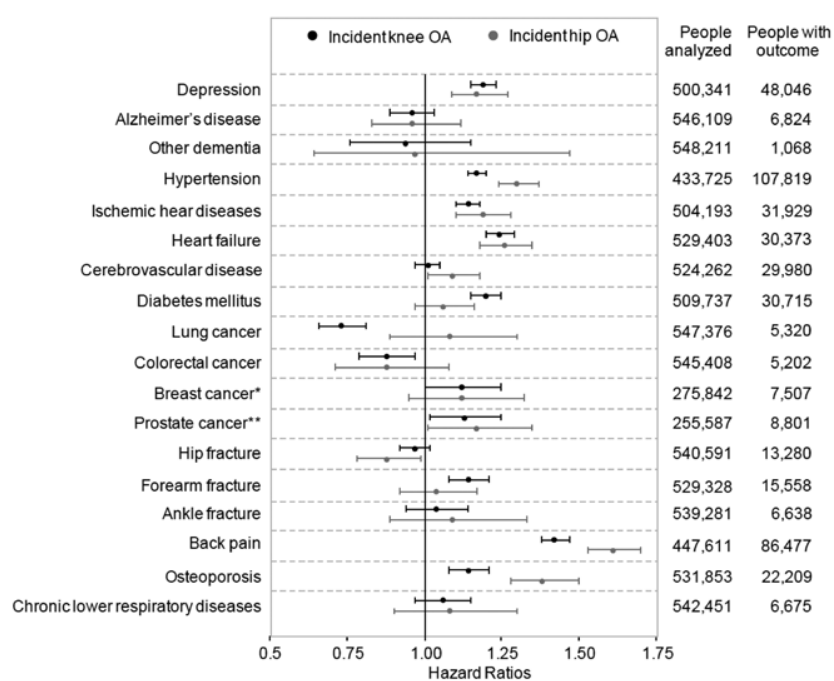

Figure 1. Adjusted hazard ratios of consultation for diseases occurring in persons with incident doctordiagnosed knee or hip OA compared to non-OA persons. "Only women included in analysis. "**nly men included in analysis.

Acknowledgments: This study was supported by FOREUM.

Disclosure of Interests: Kenneth Pihl: None declared, Aleksandra Turkiewicz: None declared, Velocity Hughes: None declared, Weiya Zhang Consultant of: Grunenthal for advice on gout management, Speakers bureau: Bioiberica as an invited speaker for EULAR 2016 satellite symposium, S.M.A. Bierma-Zeinstra Consultant of: From 2015-2017 for Infirst Healthcare, in 2019 for Phizer (consultancy fees were paid to the university), Daniel Prieto-Alhambra Grant/research support from: Professor Prieto-Alhambra has received research Grants from AMGEN, UCB Biopharma and Les Laboratoires Servier, Consultant of: DPA's department has received fees for consultancy services from UCB Biopharma, Speakers bureau: DPA's department has received fees for speaker and advisory board membership services from Amgen, Martin Englund Consultant of: Advisory Board 1 day (2019) Pfizer (Tanezumab).

DOI: 10.1136/annrheumdis-2020-eular.1315

\section{OP0185 \\ KNEE JOINT DISTRACTION IS MORE EFFICIENT IN RESTORING CARTILAGE THICKNESS THAN HIGH TIBIAL OSTEOTOMY IN PATIENTS WITH SEVERE KNEE OSTEOARTHRITIS}

M. Jansen ${ }^{1}$, S. Maschek ${ }^{2}$, R. Van Heerwaarden ${ }^{3}$, S. Mastbergen ${ }^{1}$, W. Wirth ${ }^{2}$, F. Lafeber', F. Eckstein ${ }^{2} .{ }^{1}$ University Medical Center Utrecht, Utrecht, Netherlands; ${ }^{2}$ Paracelsus Medical University, Salzburg, Austria; ${ }^{3}$ Kliniek ViaSana, Mill, Netherlands

Background: Both high tibial osteotomy (HTO) and knee joint distraction (KJD) are joint preserving surgical techniques unloading the affected femorotibial 